\title{
Мартемьянова Е.А. \\ Современные компьютерные методы анализа формы визуально воспринимаемых объектов
}

Российский государственный университет имени А.Н. Косыгина (Технологии. Дизайн.

Искусство)

(Россия, Москва)

doi: 10.18411/trnio-11-2021-59

\section{Аннотация}

Рассматриваются новые методы анализа формы визуально воспринимаемых объектов, показана степень результативности предлагаемых методов и приемов, приводятся особенности устойчивых стратегий зрительного восприятия. Показана методика компьютерной оценки формы различных объектов на базе врожденных устойчивых стратегий зрительного восприятия и вычисления количественных значений интегративных признаков, используемых человеком на начальной, скоростной фазе визуального восприятия.

Ключевые слова: системы компьютерного зрения, визуальное восприятие, структура, анализ состава и структуры, особенности зрительного восприятия.

\section{Abstract}

New methods of analyzing the shape of visually perceived objects are considered, the degree of effectiveness of the proposed methods and techniques is shown, the features of stable strategies of visual perception are given. The technique of computer evaluation of the shape of various objects based on innate stable strategies of visual perception and calculation of quantitative values of integrative features used by a person at the initial, high-speed phase of visual perception is shown.

Keywords: computer vision systems, visual perception, structure, composition and structure analysis, features of visual perception.

Известно, что именно форма несет на себе «печать времени». Мы легко отличаем один объект от другого на основании восприятия лишь очертания силуэтов или контурных изображений. Согласно словарю иностранных слов [4] форма (лат. forma) понимается двояко: во-первых, как наружный вид, внешнее очертание чего-либо. Во-вторых, как структура, воспринимаемая в большинстве случаев в виде некой системы организации, характеризующей взаиморасположение и связь её составных частей. Форма, как одно из основных изобразительных средств выражения художественного образа, всегда оставалась в поле зрения художников, архитекторов, дизайнеров. Неожиданно, в 1960-х годах, проблема анализа и идентификации формы объектов окружающей нас среды, привлекла к себе пристальное внимание математиков, инженеров, психофизиологов, организаторов производства и других специалистов. В стране необходимо было в кратчайший период времени сформировать естественно-научную базу под новую для того исторического периода «теорию распознавания образов». Фундаментальные исследования зрительного восприятия и опознания, выполненные под руководством известных отечественных ученых Б.Ф.Ломова, В.Д.Глезера, В.Ф.Рубахина, В.Ф.Венды, М.С.Шехтера, Т.П.Зинченко, позволили быстро решить основные проблемы разработок систем компьютерного зрения. Однако в процедурах оценки формы все еще оставалась противоречивая, до конца неисследованная ситуация. С одной стороны, с доисторических времен и по сей день для анализа формы внешнего вида зданий, сооружений или каких-либо других объектов применялись и применяются понятия геометрии (площадь, высота, ширина, диагональ, диаметр, периметр, толщина и т. д.). С другой стороны, при анализе формы как организации, как композиции, языка геометрии явно не хватало. Без качественно новых знаний о структуре композиции оказалось невозможным удовлетворить запросы практики, в 
частности, потребности квалиметрии, стандартизации, патентоведения, военной инженерной психологии.

К сожалению, многие разработчики систем компьютерного зрения сегодня даже не предполагают, что любая форма, воспринимаемая зрительной системой человека, обладает не только видимой, но и «скрытой» полевой структурой. Понимание и оценка полевой структуры форм приобрели в последние годы особую актуальность. Полевую структуру в некоторой степени загрубленно демонстрируют разряды молний, структуры из стальных опилок или шаров в магнитном поле, картины свечения газового разряда, возникающего вблизи поверхности объекта, помещенного в электромагнитное поле высокой напряженности (эффект Кирлиан). Более тонкие проявления так называемого «эффекта формы» давно интересуют аудиторию ученых, работающих, например, в области гальванических покрытий, в исследованиях прочности машин и механизмов, в проектировании электрических машин, в конструировании антенн радиотехнических устройств, в архитектуре и дизайне. Год от года растет число ученых, понимающих форму в физическом смысле как волновую (полевую) структуру, контуры которой совпадают с пространственными особенностями того или иного объекта. Сами объекты, из которых состоит мир, включают в себя, по крайней мере, два одинаково реальных фундаментальных физических компонента - вещество и форму, благодаря которым кусок вещества становится объектом. Практический опыт показывает, что:

— любой выступ на поверхности объекта подобен антенне, энергетическая характеристика которой может быть оценена методом газоразрядной визуализации;

- напряженность поля формы (объектов, фигур или линий) достигает максимумов по биссектрисам углов;

- единственное трехмерное тело, в виде пирамиды с квадратным основанием, в максимальной степени фокусирует энергию поля по биссекторной оси, проходящей через ее вершину;

- точки пересечения биссектрис углов формы представляют собой фокусы (лат. focus - очаг);

- кроме биссекторных направлений, максимальным уровнем энергетического потенциала обладают линии, соединяющие центры масс заряженных, намагниченных и им подобных объектов;

- любая форма образует свою волновую сигнатуру;

- приковывающие внимание наблюдателя «пустые» участки изображения могут обладать повышенной напряженностью поля, т.е. представлять собой фокусы;

- выделение зрительной системой человека видимых и невидимых (полевых) осей и фокусов - суть зрительного восприятия формы;

- в любой точке пространства форма твердого объекта может быть размягчена или разрушена волновым воздействием (эффект Хатчисона);

- формы объектов среды способны оказывать негативное воздействие на поля человеческого организма [2].

Одной из важнейших проблем, привлекающей в настоящее время пристальное внимание ученых, является проблема «автоматического» анализа формы объектов. В последние годы отечественными и зарубежными исследователями предпринимаются попытки создания информационных моделей, изоморфных на врожденные механизмы системы зрительного восприятия, позволяющих анализировать изображения, в реальном масштабе времени в условиях априорной неопределенности объектов и сцен, наблюдаемых в поле зрения. Как известно [1,3], нейрофизиологи первыми указали на наличие в зрительной системе человека двух относительно сепаратных, но тесно взаимодействующих подсистем зрения, одна из которых реализует интегративную оценку зрительной ситуации, общую 
пространственную ориентировку и локализацию стимулов, а другая - тонкий детальный анализ. Зрительный образ у человека является продуктом взаимодействия обеих этих субсистем.

В новой зрительной ситуации первой включается система интегративной оценки. При этом зрительная система работает в скоростном режиме функционирования. Информация, получаемая в скоростном режиме, крайне важна для наблюдателя, так как происходит оценка степени опасности попавшего в поле зрения объекта. Вторая субсистема работает в режиме тонкого детального анализа, требующего значительных временных затрат. В отличие от скоростного, интегративного режима работы, детальный анализ осуществляется с помощью движений глаз.

Для создания «человекоподобных» систем компьютерного зрения, современная номенклатура понятий (взятых в основном из аналитической геометрии, алгебры изображений или других, связанных с математикой научных дисциплин) оказалась неэффективной, так как все разработчики копируют лишь фазу детального анализа.

В настоящее время автором были созданы ряд компьютерных алгоритмов и программ систем компьютерного зрения на базе врожденных устойчивых стратегий зрительного восприятия и вычисления количественных значений интегративных признаков, используемых человеком на начальной, скоростной фазе визуального восприятия.

Главная цель проделанной работы - существенное сокращение количества исходных данных при максимальном снижении вычислительной трудоемкости оценки формы.

С целью верификации разработанных алгоритмов, ряда программных модулей и окончательной программы, получившей название «Анализатор - М», объектом изучения были различные возрастные группы испытуемых общей численностью 3800 человек (дошкольники 3-5 лет, школьники 8-11 лет, студенты 18-20 лет, специалисты различных областей 25-45 лет). Вместе с этим, объектом изучения были группы людей различных специальностей одного возраста (архитекторы, инженеры-конструкторы, художникиоформители). В исследованиях использовались следующие методики: видеосъемка, предъявление изображений на экранах переносных и стационарных тахистоскопов, электроокулография (рис.1). Проведенные тахистоскопические эксперименты в предпороговой зоне зрительного восприятия подтвердили фазность этого процесса. Попавший в поле зрения объект вначале воспринимается как пятно. Для оценки опасности на этой фазе зрительная система мгновенно включает все рецептивные поля, все каналы переработки информации, характеризующие форму, цвет, ориентацию, размеры и местоположение, сигналы с которых порождают интегративные признаки. Первый интегративный признак, характеризующий силу энергетического воздействия на сетчатку глаз попавшего в поле зрения объекта, наблюдаемого вначале в виде бесформенного пятна, получил название «визуальная масса» [5]. На последующем этапе восприятия начинает проявляться вначале остов, а затем «скелет» формы. При этом происходит оценка еще трех признаков, получивших названия: «степень динамичности визуальной массы» (характеризует степень концентрации визуальной массы по различным направлениям); «вектор динамичности визуальной массы» (характеризует направление устремленности визуальной массы в поле зрения); угол наклона «главной динамической оси», вдоль которой сконцентрировано основное количество визуальной массы (характеризует ориентацию наблюдаемого объекта). Структура воспринимаемого изображения как бы «лепится» из визуальной массы. В результате оказалось, что уже на начальной стадии скоростного анализа система компьютерного зрения способна идентифициовать загрубленные (или зашумленные) объекты.

Bce перечисленные интегративные (психофизиологические) признаки, за исключением угла наклона главной динамической оси, измеряются в относительных единицах. В основу машинной оценки количества визуальной массы автором была положена подтвержденная многочисленными экспериментами следующая функциональная зависимость: 


$$
\mu=f(k ; l ; \rho ; \alpha ; \lambda ; s ; x ; y ; z),
$$

где $k$ - коэффициент, учитывающий особенности зрительного восприятия, $l$ - периметр, $\rho$ коэффициент отражения, $\alpha$ - угол наклона главной динамической оси объекта относительно принятых осей координат, $\lambda$ - доминирующая длина волны, $s$ - площадь, $x, y, z$ - координаты пикселей изображения.
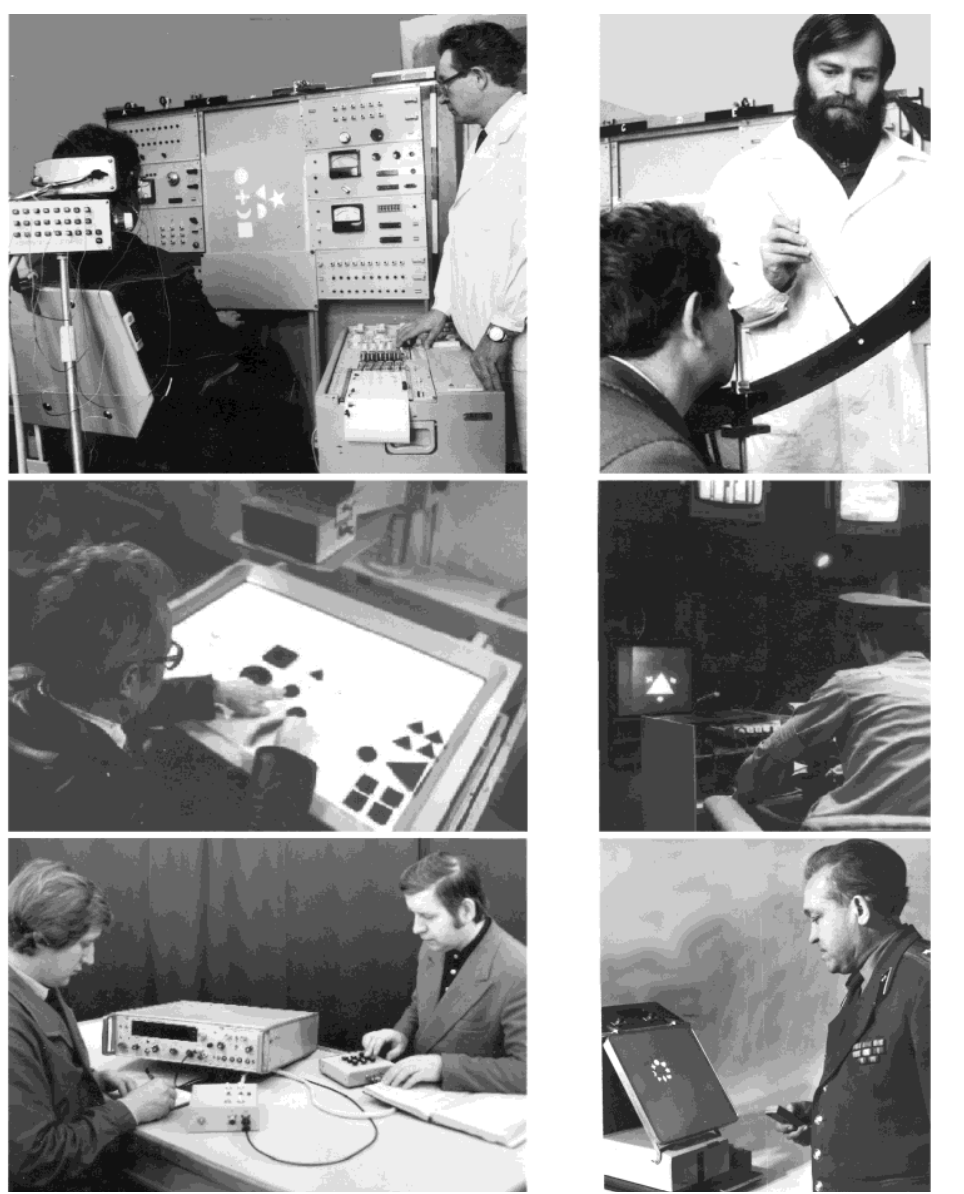

Рисунок 1. Исследования зрительной оченки форм в лабораторных условиях и на рабочих местах

В проведенных исследованиях в качестве тест-объектов использовались плоские полихроматические фигуры, фрактальные и линеарные плоскостные композиции, фотоизображения трехмерных объектов.

Эксперименты показали, что внимание наблюдателя акцентируется на определенных участках изображения даже в тех случаях, когда изучаемые композиции имеют относительно сложную структуру. При этом, главными значимыми центрами структуры наблюдатель считает:

- области пересечения большего количества осей;

- места пересечения главных динамических осей;

- при прочих равных условиях, центры, расположенные в левом верхнем квадранте изображения;

- области изображения, обладающие большей визуальной массой;

- выпуклые участки контуров, а среди них участки с большей степенью динамичности.

На завершающем этапе исследований разработанная автором компьютерная программа (рис. 2), получившая название «Анализатор М», позволяет:

— вычислять значения визуальной массы пикселей любой цветности; 
- формировать на экране монитора трехмерный «энергетический ландшафт» анализируемого изображения, вращать его во всех направлениях с целью детального осмотра со всех сторон;

- вычислять и обозначать осями координат местоположение центров масс кластеров или элементов и накладывать оси на их изображение;

- вычислять величину визуальной массы наблюдаемых объектов, кластеров или отдельных элементов;

- вычислять значение степени динамичности и величину вектора динамичности наблюдаемых объектов, явлений (подобным хвостам комет, северным сияниям) с замкнутыми или разомкнутыми контурами, меняющими форму во времени;

вычислять направление главной динамической оси фрактальных изображений (типа изменяющихся с течением времени: разливов рек, перемещений пожаров, трансформаций сети дорог) и изображать его на анализируемой композиции;

- вычислять местоположение оси баланса масс и изображать ее на экране;

- выделять анализируемые объекты из среды и ранжировать их по уровням восприятия;

- формировать кортежи зрительного восприятия.

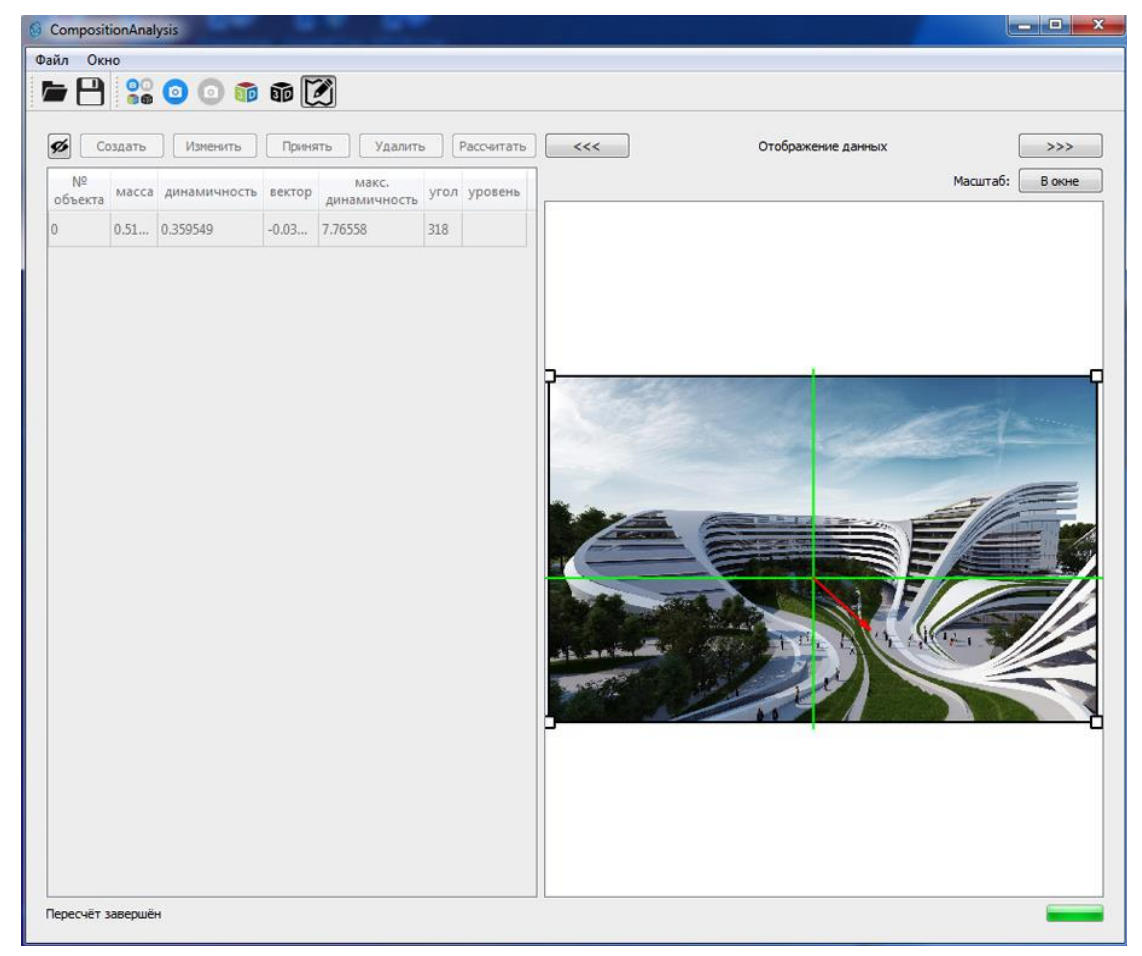

Рисунок 2. Окно программы «Анализатор - М»

$* * *$

1. Иваницкий, А. М. Мозговые механизмы оценки сигналов // А. М. Иваницкий. - Москва: Медицина, 1976. 264 с.

2. Лимонад, М.Ю. Живые поля архитектуры // М.Ю. Лимонад, А.И. Цыганов. - Обнинск: Титул, 1997. - С. 77.

3. Митькин, А.А. Дискуссионные аспекты психологии и физиологии зрения // А.А.Митькин // Психологический журнал. Т.3.- 1982.- №1.- С.31-42.

4. Словарь иностранных слов // под ред. И.В.Лехина, С.М.Локшиной, Ф.Н.Петрова, Л.С.Шаумяна. - Москва: Совет. энцикл., 1964. - 784 с.

5. Шаповал, А. В. Разработка технических и программных средств для количественной оценки формы бинарных изображений // А. В. Шаповал // Приволжский научный журнал. - Н. Новгород, 2007. - № 4. - С. $38-53$. 